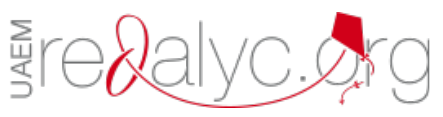

Centro Sur

ISSN: $2600-5743$

compasacademico@icloud.com

Grupo Compás

Ecuador

\title{
Plan estratégico para el centro de informática orientado a la propagación de las tecnologías de la información y la comunicación
}

Ponce Chalén, Verónica Cecilia

Plan estratégico para el centro de informática orientado a la propagación de las tecnologías de la información y la comunicación

Centro Sur, vol. 2, núm. 1, 2018

Grupo Compás, Ecuador

Disponible en: http://www.redalyc.org/articulo.oa?id=588861692003 


\title{
Plan estratégico para el centro de informática orientado a la propagación de las tecnologías de la información y la comunicación
}

\author{
Strategic plan for the computer center aimed at the \\ propagation of information and communication technologies \\ Verónica Cecilia Ponce Chalén veroc_05@hotmail.com \\ Universidad de Guayaquil, Ecuador \\ http://orcid.org/0000-0003-2837-9692
}

Centro Sur, vol. 2, núm. 1, 2018

Grupo Compás, Ecuador

Recepción: 17 Enero 2017 Aprobación: 24 Julio 2018

Redalyc: http://www.redalyc.org/ articulo.oa?id=588861692003
Resumen: El estudio se realizó en la Universidad Estatal Península de Santa Elena - UPSE, que consiste en diagnosticar el nivel de conocimiento que están estudiante, trabajadores y profesionales internos y externos en el uso de las Tecnologías de la Información y la Comunicación - TIC. Con esta diagnóstico fortalecer capacidades mediante la gestión estratégica del Centro de Capacitación de Informática de la Facultad de Sistemas y Telecomunicaciones, que incluya módulos de capacitación y formación continua en Tecnologías de la Información y Comunicación y tópicos de Economía y Negocios, orientado al cumplimiento de la responsabilidad de la escuela en el ámbito de la autogestión y enmarcado en el respaldo a la gestión institucional en la región, en términos de mejorar el desempeño profesional.

Palabras clave: TIC, Capacitación, Formación Continua, Gestión Institucional.

Abstract: The study was carried out at the Santa Elena Peninsula State University UPSE, which consists in diagnosing the level of knowledge that students, workers and internal and external professionals are in the use of Information and Communication Technologies - ICT. With this diagnosis, strengthen capacities through the strategic management of the Computer Training Center of the Faculty of Systems and Telecommunications, which includes training and continuous training modules in Information and Communication Technologies and topics of Economics and Business, aimed at compliance with the The responsibility of the school in the field of selfmanagement and framed in supporting institutional management in the region, in terms of improving professional performance.

Keywords: ICT, Training, Continuing Education, Institutional Management.

\section{INTRODUCCION}

La Universidad Estatal Península de Santa Elena, como entidad educativa llamada a ser un referente del avance de la ciencia y la tecnología en la región, se mantiene en la búsqueda constante de maneras para mejorar el nivel de consolidación de su actuación en cada uno de los pilares fundamentales que conforman la universidad de hoy, vale decir, la investigación, la gestión, la docencia y la vinculación con la comunidad.

Como parte de su misión y en cumplimiento de las recomendaciones que efectuara en su oportunidad el Consejo Nacional de Evaluación y Acreditación del Sistema de Educación Superior, la Universidad de Santa Elena ha elaborado un plan de mejoramiento orientado a satisfacer los requerimientos que en el marco de su desarrollo como entidad de 
educación superior, se le exigen, por parte no sólo del gobierno, sino de la sociedad en su conjunto.

En este contexto, la Facultad de Sistemas y Telecomunicaciones, y en específico la Escuela de Informática, comprometida con el logro de los objetivos institucionales y el cumplimiento de los indicadores a ser evaluados en el marco de su proceso de acreditación, ha trabajado en el desarrollo, integración y aplicación de los mecanismos que garanticen la obtención de la certificación de sus niveles de calidad, el respaldo a la gestión institucional y al desarrollo de la sociedad, y su propia permanencia en el tiempo.

En los últimos años se ha evidenciado la gran importancia de las tecnologías de información y las comunicaciones en diario vivir del Ecuador y del mundo, teniendo un mayor impacto en las Universidades, siendo estas un referente integral. En la actualidad, los requerimientos respecto al uso de TICs es fundamental, por ejemplo para la obtención de puntos para concurso de méritos es necesaria la capacitación en estos tópicos, además la evolución de aplicaciones web, para móviles hace que se necesite este tipo de conocimiento inclusive como una alternativa para hacer negocios. En este se hace fundamental la planificación para que el centro de informática pueda tener estrategias de sostenibilidad y que se convierta en un brazo de autogestión para la UPSE.

\section{MATERIALES Y MÉTODOS}

Dentro de la metodología se plantearon dos encuestas, la primera a personal de la Universidad entendidos en la temática para medir el conocimiento respecto al centro y la importancia de la planeación estratégica, la segunda se realizó a potencial mercado objetivo como lo son estudiantes, profesionales internos y externos y medir la percepción respecto a los servicios del centro.

Población y muestra

\begin{tabular}{|l|l|l|}
\hline Elementos & Ni & $\begin{array}{l}\text { Muestreo } \\
\text { por } \\
\text { Criterios }\end{array}$ \\
\hline $\begin{array}{l}\text { personal de la } \\
\text { Universidad( } \\
\text { docentes, } \\
\text { administrativos } \\
\text { diferentes } \\
\text { áreas) }\end{array}$ & 300 & 35 \\
\hline
\end{tabular}

Los Autores 


\begin{tabular}{|l|l|l|}
\hline Elementos & $\mathrm{Ni}$ & $\begin{array}{l}\text { Muestreo } \\
\text { por } \\
\text { criterios }\end{array}$ \\
\hline $\begin{array}{l}\text { estudiantes, } \\
\text { profesionales } \\
\text { internos y } \\
\text { externos }\end{array}$ & 6500 & 140 \\
\hline
\end{tabular}

La percepción que se tiene, de acuerdo al estudio de tipo descriptivo que se realizó en la universidad es la siguiente. Se realizaron 140 encuestas derivadas de la siguiente fórmula:

$$
n=\frac{n Z^{2} p q}{e^{2}(N-1)+Z^{2} p q}
$$

Tabla de descripción de la muestra

\begin{tabular}{|l|l|}
\hline$N$ & 6500 \\
\hline $\mathbf{P}$ & 0,92 \\
\hline $\mathbf{Q}$ & 0,08 \\
\hline $\mathbf{E}$ & $5 \%$ \\
\hline $\mathrm{Z}$ & $\mathbf{1 , 9 6}$ \\
\hline $\begin{array}{l}\mathbf{n} \\
\text { inicial }\end{array}$ & $\mathbf{1 4 0}$ \\
\hline n final & $\mathbf{1 4 0}$ \\
\hline
\end{tabular}

Los autores

Con los datos obtenidos se diseñó una matriz FODA y a partir de esta se generaron las matrices de estrategias cruzadas Fortalezas Oportunidades, Debilidades - Oportunidades, Fortalezas - Amenazas y Debilidades - Amenazas.

Con estos resultados obtenidos, se diseñó la propuesta del Plan Estratégico para el Centro de Cómputo de la UPSE respecto a la propagación de la Tecnologías de la Información y la Comunicación

ü Las variables examinadas son las siguientes:

ü Equipamiento del hogar

ü Uso de computadora

ü Uso del Internet

ü Tenencia de celular

ü Teléfono celular inteligente

ü Perfil de usuario de redes sociales

Dentro de los datos más relevantes obtenidos esta que en el 2012 se reportaron 839.705 usuarios de teléfonos inteligentes (Smartphone), un $60 \%$ más que lo del 2011, cuando llegó a 522.640 usuarios, según los últimos datos de la encuesta de Tecnologías de la Información y la Comunicación (TIC) del Instituto Nacional de Estadística y Censos (INEC). El estudio, que se realizó en diciembre de 2012, se hizo en 21.768 
hogares a personas de 5 años y más, a nivel nacional, regional, provincial, de nivel urbano y rural.

Según esta encuesta, en el 2012 el 12,2\% de las personas que tienen un celular poseen un teléfono inteligente (Smartphone) frente al $8,4 \%$ registrado en el 2011.

En Ecuador existen 6'859.938 personas que tienen al menos un celular activado, un $10 \%$ más que lo registrado en el 2011 y representa al $50,4 \%$ de la población de 5 años en adelante. El 52,6\% de los hombres tiene teléfono celular activado, frente al 48,3\% de las mujeres. El grupo etario con mayor uso de teléfono celular activado es la población que se encuentra entre 25 y 34 años con el 77,6\%, seguido de los de 35 a 44 años con el 72,8\%.

Otras de las variables de estudio es el índice de penetración de las TIC y para esto se multiplicaron los pesos relativos por las variables de los hogares, y, se sumaron de todas las variables ya multiplicadas por los pesos. La jerarquía utilizada es la siguiente:

1. acceso y uso de internet.

2. medios o herramientas para acceder al internet.

3. tecnología.

Este indicador se interpreta bajo las siguientes condiciones:

ü Hogares que tienen la sumatoria de 0 hasta 34, se encuentran en la categoría de baja penetración (categoría 1 ).

ü Hogares que tienen la sumatoria de 34 hasta 68, se encuentran en la categoría de penetración mediana (categoría 2 ).

ü Hogares que tienen la sumatoria de 68 hasta 100, se encuentran en la categoría de alta penetración (categoría 3).

\section{RESULTADOS}

Utilizando la información del FODA, de las matrices cruzadas se determinaron los siguientes resultados.

3.1. Facilitar y mejorar el proceso de aprendizaje en TICS para el mejoramiento de los procesos actuales de enseñanza y aprendizaje e incentivando la investigación.

ü Proveer herramientas fáciles de usar y apoyar, con las funciones necesarias para asistir en el proceso de aprendizaje y enseñanza.

ü Incrementar el uso de las TICs en proceso enseñanza y aprendizaje.

ü Promover la innovación educativa en la aplicación y uso de tecnologías actuales.

ü Promover ambientes que coadyuven el desarrollo de investigación para los estudiantes, profesionales de la UPSE y externos.

ü Adquirir o desarrollar tecnologías que impacten las distintas metodologías de enseñanza en la UPSE. 
Propuesta de Plan Estratégico para el centro de informática 2016-2020

\begin{tabular}{|c|c|c|c|}
\hline OBBETWO ESTRATEGCOO & OBEETNO OPERATNO & NDOC. & PRESSPPESTO \\
\hline \multirow{3}{*}{ 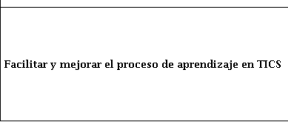 } & & 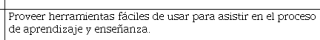 & 2500000 \\
\hline & 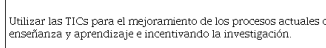 & 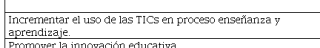 & 25000000 \\
\hline & & 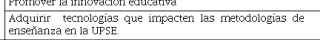 & $\begin{array}{l}5300000 \\
55600,00\end{array}$ \\
\hline
\end{tabular}

Los autores

3.2 Mantener y mejorar la infraestructura destinada al centro de informática en buen estado, segura y con tecnología de punta.

Infraestructura tecnológica en capacidad de proveer acceso integrado a todos los recursos tecnológicos necesarios para los estudiantes, profesionales internos y externos de la UPSE. Innovando y realizando estudios de mercados validando las necesidades de capacitación.

ü Mejoramiento de la infraestructura e insumos informáticos a través de la actualización y asignación de recursos eficientes con el fin de extender y diversificar las capacidades, servicios y apoyo que brindará en centro asegurando la calidad de servicios.

ü Crear infraestructura inalámbrica adecuada manteniendo consistencia con las políticas de acceso y buen uso de redes además estar acordes con los lineamientos de acreditación universitaria.

ü Identificación de oportunidades para proveer aplicaciones para móviles, licencias de aplicaciones, desarrollo de procesos para la adquisición de licencias por volumen.

Propuesta de Plan Estratégico para el Centro de Informática 2016-2020

Los autores

3.3. Difundir e incrementar el conocimiento en las tecnologías de información y comunicación.

Asegurar que la facultad de informática y telecomunicaciones, estudiantes, profesionales internos y externos de la UPSE, utilicen con fluidez las tecnologías que son básicas y de especialidad.

ü Revisar constantemente los estándares de calidad de la UPSE, respecto a competencias tecnológicas para asegurar que están acordes con las necesidades y expectativas del mercado objetivo interno y externo.

ü Facilitar la participación para establecer metas específicas en conocimiento de las TICs a través de reuniones, encuentros, reconociendo la diversidad tecnológica entre los diferentes grupos de atención.

ü Ampliar la creación de programas de apoyo a las aplicaciones para ayudar alcanzar las metas de la UPSE, mejorando así indicadores de aseguramiento y calidad de la educación. 
ü Instruir a todos los usuarios en como existir de forma segura en TICS, además de aplicaciones.

Propuesta de Plan Estratégico para el Centro de Informática 2016-2020

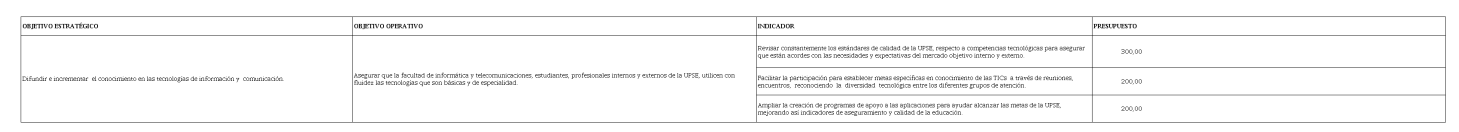

Los autores

\section{DISCUSIÓN}

Existen elementos teóricos que evidencian las importancias de la planeación estratégica en los procesos de gestión en este caso para el centro de informática. Respecto a los resultados, en las dos encuestas, en ambas se evidencia la importancia de la planificación de centro de informática, además se pudo evidenciar los potenciales cursos a dictarse. En lo que respecta al plan estratégico, este posee 5 grandes ejes, se realizó la planificación para 5 años ampliando así los potenciales servicios a emplearse La planeación estratégica se vuelve fundamental para poder tener claro el horizonte del centro de informática y que este se torne en importante para captar fondos para la UPSE.

\section{Referencias}

[1] Area Moreira, M. (2009). Introducción a la tecnología educativa. España: Universidad de La Laguna

[2] Area Morerira, M., Gros Salvat, B. y Marzal García-Quismondo. M.A. (2008). Alfabetizaciones y tecnologias de la información y la comunicación. Madrid, España: Síntesis

[3] Balagué, F. \& Zayas, F. (2007). Usos educatius dels blogs. Recursos, orientacions $i$ experiencies per a docents. Barcelona, España:UOC

[4] Barba, C., \& Capella, S. (2010). Ordenadores en las aulas. La clave es la metodología. Barcelona,España: Graó

[5] ARANDA, A. (2007). Planificación estratégica educativa. Segunda edición. Quito, Ecuador: Ediciones Abya Yala. 